\title{
EDUCAÇÃO EM SAÚDE: UMA ESTRATÉGIA PARA MINIMIZAR O ABANDONO DO TRATAMENTO DA TUBERCULOSE EM PACIENTES DO INSTITUTO DE PESQUISA CLÍNICA EVANDRO CHAGAS/FIOCRUZ
}

\section{HEALTH EDUCATION: A STRATEGY TO MINIMIZE THE ABANDONMENT OF TREATMENT OF TUBERCULOSIS PATIENTS IN CLINICAL RESEARCH INSTITUTE EVANDRO CHAGAS THE OSWALDO CRUZ FOUNDATION}

\author{
Simone Carvalho Neves ${ }^{1}$, Valéria Cavalcanti Rolla ${ }^{2}$, Claudia Teresa Vieira de Souza $^{3}$ \\ 1,2 Laboratório de Pesquisa Clínica em Micobacteriose - Tuberculose do Instituto de Pesquisa Clínica \\ Evandro Chagas - Fundação Oswaldo Cruz. simone.carvalho@ipec.fiocruz,br; \\ valeria.rolla@ipec.fiocruz.br \\ ${ }^{3}$ Docente do Programa de Pós-Graduação em Ensino em Biociências e Saúde/Instituto Oswaldo \\ Cruz/Fundação Oswaldo Cruz; Pesquisadora titular do Laboratório de Epidemiologia Clínica do Instituto \\ de Pesquisa Clínica Evandro Chagas/Fundação Oswaldo Cruz \\ Avenida Brasil 4365 - Manguinhos CEP: 21045-900 -Rio de Janeiro - Brasil. Tel. 021 2260-9749/3865- \\ 9624. claudia.souza@ipec.fiocruz.br
}

\section{RESUMO}

A tuberculose ainda é um grave problema de Saúde Pública no Brasil. O tratamento irregular e o abandono são considerados os maiores entraves para o controle e a eliminação desta doença. $\mathrm{O}$ estudo pretendeu identificar fatores sócio-demográficos e comportamentais que influenciaram os pacientes a abandonarem o tratamento a fim de implantar um Programa de Educação em Saúde no Instituto de Pesquisa Clínica Evandro Chagas da Fundação Oswaldo Cruz. Foi utilizado um roteiro semi-estruturado nas entrevistas. A análise foi baseada no Discurso do Sujeito Coletivo. Foram entrevistados 17 pacientes que abandonaram o tratamento no período de 2000-2006. Os motivos do abandono do tratamento: tomar os remédios em jejum, a quantidade de comprimidos e cápsulas e os efeitos adversos. Esperamos que novas iniciativas em educação em saúde possam surgir para o controle da tuberculose.

Palavras-chave: Abandono de Tratamento, Tuberculose, Educação em Saúde.

\begin{abstract}
Tuberculosis is still a serious problem for the Public Health in Brazil. The irregular treatment and the abandon are considered the large problems for the control and elimination of this disease. The present study intended to identify those socialdemographic and behavioral factors which caused influence in the patients to abandon the tuberculosis treatment in order to implement a program of health education in the Clinical Research Institute Evandro Chagas at the Oswaldo Cruz Foundation. We utilized a script semi-structured interview. The analysis of the interview was based in the technique of the Collective Subject Discussion of the Subject. We interviewed 17 patients that abandoned treatment in the period 2000-2006. The reasons for the abandonment of treatment: take medicine on an empty stomach, the amount of tablets and capsules and adverse effects. We hope that new initiatives in health education may arise for the control of tuberculosis.
\end{abstract}

Key-words: Tuberculosis, Treatment Abandon, Health Education. 


\section{Introdução}

A tuberculose (TB) é ainda um grave problema de Saúde Pública no Brasil e tem chamado atenção também nos países desenvolvidos, como os Estados Unidos e Japão, onde se pensava que esta doença estaria sob controle. De fato, nas últimas décadas a freqüência de casos confirmados de tuberculose tem aumentado (Rubel \& Garro, 1992; Ruffino-Netto, 2002; Ministério da Saúde, 2004; WHO, 2004).

A TB é uma doença infecciosa causada pelo Mycobacterium tuberculosis que foi descoberta em 1882, por Robert Koch, e sinalizada como um problema mundial somente em 1993 (WHO, 2004). A transmissão da TB pode ocorrer por três formas: inalação de aerossóis que contenham o bacilo, ingestão de alimentos contaminados e por inoculação direta (Ministério da Saúde, 2002).

De acordo com relatório da Organização Mundial da Saúde, a TB foi a principal causa de morte na Europa e Estados Unidos até o início do século XX e mesmo com o advento dos tuberculostáticos, esta doença infecciosa continua causando morte nas pessoas com idade produtiva em todo o mundo (WHO, 2004).

No final da década de 80, com o surgimento da epidemia do HIV, os casos de tuberculose aumentaram, sendo este o maior fator de risco para o adoecimento dos indivíduos infectados pelo HIV (Ministério da Saúde, 2002).

O abandono do tratamento da TB favorece o desenvolvimento de bacilos multirresistentes, tornando a terapia mais complexa e mais longa com o emprego de um número maior de drogas, e que conseqüentemente aumentam os efeitos adversos e favorecem a falta de estímulo desses indivíduos para levar o tratamento até o fim (Sumartojo, 1993).

Estima-se que existam aproximadamente 1200 casos de bacilos multirresistentes a drogas (MDRTB) em tratamento, 90\% deles nos estados de São Paulo e Rio de Janeiro. Os casos de MDRTB ocorrem como resultado do tratamento irregular, como: a falta de adesão à terapia, poucos médicos e enfermeiros treinados e que fazem prescrições de forma incorreta e/ou devido a problemas fisiológicos, tais como a interação medicamentosa ou a má absorção dos medicamentos (Drobniewski \& Balabanova, 2002).

As estratégias utilizadas para o controle da TB vêm sendo realizadas através de ações preconizadas pelo Ministério da Saúde que são: o diagnóstico e tratamento precoce dos indivíduos doentes, além de busca ativa de sintomáticos respiratórios 
através do Programa da Saúde da Família (PSF) e quimioprofilaxia de contatos de casos bacilíferos quando indicado (Ministério da Saúde, 2002).

A estratégia do tratamento diretamente supervisionado, tradução da expressão "directly observed short course therapy", comumente denominada DOTS, foi adotada oficialmente pelo governo brasileiro em 1998, com o objetivo de diminuir o abandono e aumentar o controle da TB (Netto, Dye, Raviglione, 1999). No entanto, até os dias atuais, a cobertura do tratamento diretamente supervisionado ainda é baixa, aumentou de 25\% em 2002 para 34\% em 2003 (Ministério da Saúde, 2004). A estratégia de expandir o tratamento diretamente supervisionado no Brasil foi limitada, não produzindo os efeitos almejados em relação à diminuição do abandono de tratamento de TB (WHO, 2004).

De fato, vários são os fatores que influenciam a adesão ao tratamento, podendo ser sócio-demográficos e comportamentais: idade, sexo, alcoolismo, tabagismo, infecção pelo HIV, pobreza ou miséria, desemprego, uso de drogas e poucos anos de estudo. Fatores associados aos problemas clínicos: os efeitos adversos da medicação, freqüência diária ou intermitente do uso de medicamentos, tipo de supervisão de tomada das drogas, tempo de duração do tratamento, forma clínica da doença, resistência às drogas tuberculostáticas. Em relação aos recursos humanos: a falta de estrutura de atendimento (serviços de saúde ainda mostram-se pouco capacitados para atender e assistir as necessidades básicas dos pacientes tuberculosos).

Além disso, pode-se mencionar: capacidade de oferecer as consultas periodicamente em duração e em números adequados para um tratamento, abordagem multidisciplinar do paciente, estratégia de recuperação dos faltosos, incentivo financeiro à adesão, capacidade de atender a demanda local, disponibilidade de laboratório para o diagnóstico precoce da TB e acompanhamento dos pacientes em tratamento, entre outros (Braga, Hijjar, \& Procópio, 2004; Drobniewski \& Balabanova, 2002; Ministério da Saúde, 2002).

Diante deste panorama desafiador, identificamos insuficiente conscientização e entendimento dos pacientes, em sua maioria, sobre a TB. Trata-se de uma questão importante que apresenta múltiplas conseqüências e implicações adversas se não houver intervenção por parte das autoridades públicas.

Contudo, uma pesquisa que vise obter subsídios para novas iniciativas e estratégias de controle da TB é necessária para uma intervenção eficaz; processo este 
que almeja minimizar o abandono do tratamento de TB de maneira que a educação em saúde seja a ferramenta principal a ser utilizada neste contexto.

Acreditamos que a estratégia de implantação de um programa de educação em saúde direcionado a pacientes de TB seria uma alternativa para minimizar o abandono.

\section{Método}

A pesquisa foi realizada no Centro de Clínicas Adrelírio Rios Gonçalves onde funciona o Laboratório de Pesquisa Clínica em Micobacteriose do Instituto de Pesquisa Clínica Evandro Chagas (LAPCLIN TB IPEC-FIOCRUZ), Rio de Janeiro. Trata-se de um estudo descritivo, com abordagem qualitativa. A escolha da abordagem qualitativa justifica-se, porque a pesquisa teve a intenção de ouvir a fala de alguns pacientes que abandonaram o tratamento da $\mathrm{TB}$, tentando compreender e identificar os fatores que os levaram a este desfecho.

Este estudo foi apresentado ao Colegiado Técnico Científico (CTC) e aprovado pelo Comitê de Ética em Pesquisa (CEP) do IPEC (protocolo n ${ }^{\circ}$ 0010.0.009.00-06). Todos os sujeitos da pesquisa foram convidados e informados sobre o interesse, os objetivos, os possíveis benefícios e aplicabilidade do estudo e assinaram o Termo de Consentimento Livre e Esclarecido (TCLE), respeitando as questões éticas de pesquisas envolvendo seres humanos, preservando a privacidade dos entrevistados e a confidencialidade das informações obtidas.

Os sujeitos pesquisados foram os pacientes incluídos no LAPCLIN TB que abandonaram o tratamento de TB de 2000 a 2006, neste período foram diagnosticados 456 pacientes com TB, $339(74,3 \%)$ tiveram alta por cura e 83 pacientes $(18 \%)$ abandonaram o tratamento de TB. Todos os 83 pacientes que abandonaram o tratamento da TB foram convidados para participar da pesquisa.

Foi considerado abandono de tratamento o paciente que não compareceu à consulta agendada e/ou que deixou de tomar os medicamentos ao menos por 30 dias consecutivos (Ministério da Saúde, 2004).

Utilizamos como critério de inclusão todos os pacientes que tiveram diagnóstico confirmado ou diagnóstico clínico da TB e que posteriormente abandonaram o tratamento. O critério de exclusão foi considerado todos pacientes que não puderam ser localizados devido à mudança de endereço e/ou telefone.

O recrutamento para a realização das entrevistas foi realizado através do contato por telefone e/ou carta solicitando o comparecimento do paciente para participar da 
pesquisa e para sensibilizá-lo a retornar ao tratamento da TB. A título de incentivo foi fornecido custeio para um lanche e transporte para os pacientes que compareceram a entrevista agendada.

A pesquisa foi realizada a partir de um roteiro de entrevista com 6 (seis) perguntas semi-estruturadas que oferece ao pesquisador uma flexibilidade maior, levando em consideração que permite intervenções (Minayo, Deslandes \& Gomes, 2002). Todas as entrevistas foram gravadas e posteriormente as falas transcritas em sua totalidade.

Para a análise dos dados foi utilizada à técnica do Discurso do Sujeito Coletivo. Trata-se de uma proposta de organização e tabulação de dados qualitativos de natureza verbal, obtido através dos depoimentos. Essa técnica confere naturalidade, espontaneidade e vivacidade ao pensamento coletivo, o que contrasta fortemente com as formas clássicas de apresentação de resultados qualitativos, visando assim, consolidar os discursos individuais, extraindo-se as expressões-chave e idéias centrais de modo que expressem ao final um pensamento de uma coletividade (Lefévre \& Lefévre, 2003).

\section{Resultados e Discussão}

Desenvolver um estudo sobre abandono de tratamento é um desafio, devido aos inúmeros fatores sócio-comportamentais que influenciam na adesão ao tratamento, como já mencionamos anteriormente. Mediante observação das próprias características dos pacientes que abandonaram o tratamento da TB, sabíamos da dificuldade em recrutar este grupo para a realização das entrevistas, principalmente devido ao tempo decorrido entre a última consulta e a realização desta pesquisa, em média quatro anos.

No período de 2000-2006, 83 pacientes abandonaram o tratamento da TB, desses apenas 17 (20,5\%) compareceram para as entrevistas realizadas no período de julho de 2006 a fevereiro de 2007, os mesmos foram encaminhados para avaliação médica para possível retratamento ou não da doença após serem entrevistados.

As principais justificativas do não comparecimento à entrevista foram a não localização por mudança de endereço e/ou de telefone $(23,0 \%)$ e o desinteresse dos pacientes que receberam correspondência e/ou telefonema para comparecer ao LAPCLIN TB para a pesquisa $(56,6 \%)$.

Todos os entrevistados que compareceram ao IPEC se disponibilizaram em participar da pesquisa e assinaram o TCLE. Os depoimentos obtidos permitiram 
identificar o entendimento dos pacientes em relação à $\mathrm{TB}$, as suas concepções e os mitos em relação a esta doença.

Doze dos pacientes entrevistados eram do sexo masculino, com idade entre $22 \mathrm{e}$ 58 anos, todos em na faixa etária produtiva.

Pesquisadores que trabalham com TB constataram que o abandono do tratamento é mais freqüente em pacientes do sexo masculino, grupo este em que predomina o desemprego e o alcoolismo (Deheizelin et al., 1996; Albuquerque et al., 2001; Braga, Hijar \& Procópiio, 2004; Bergel \& Gouveia, 2005; Nogueira et al., 2006).

Em relação ao estado civil, dos doze homens, seis informaram estar casados. Além disso, observamos dos dezessete pacientes entrevistados, nove (53\%) relataram não ter nenhuma renda mensal e os demais informaram ter de um a dois saláriosmínimos. Em relação à escolaridade, do total de entrevistados, 10 (58,8\%) entrevistados relataram ter menos de oito anos de escolaridade. Identificamos ainda que 14 pacientes $(82,4 \%)$ apresentaram sorologia anti-HIV positiva, sendo que destes, oito tiveram o diagnóstico de TB pulmonar, dois TB disseminada e uma com TB cutânea.

Entrevistamos cinco mulheres, com idade entre 28 e 43 anos, das quais três informaram ser solteiras. Em relação à renda familiar, as entrevistadas também apresentaram renda inferior a 2 salários mínimos.

Podemos observar nos pacientes entrevistados que 10 (58,8\%) apresentaram-se como tabagistas ou com história prévia de tabagismo, sendo 7 destes do sexo masculino.

Podemos verificar de acordo com os resultados apresentados que o perfil clínicoepidemiológico dos pacientes entrevistados, apesar de ser uma amostra pequena, é similar aos descritos na literatura, ou seja, a pouca capacidade de obter uma renda mensal e a baixa escolaridade são fatores associados ao abandono do tratamento da TB (Tangüis et al., 2000).

Conforme descrito anteriormente, utilizamos para a análise, a técnica do Discurso do Sujeito Coletivo (DSC), ou seja, recorremos à "organização e tabulação dos dados qualitativos de natureza verbal”. A partir das seis perguntas do roteiro de entrevista, organizamos um quadro esquemático com as figuras metodológicas: Ideias Centrais (IC) e Expressões-Chave $(\mathrm{ECH})$ para descrever o DSC (Lefévre \& Lefévre, 2003).

As expressões-chave, selecionadas a partir das entrevistas realizadas com o paciente, e a ideia central, que traduziu o sentido da fala, permitiu a construção dos DSCs que "revelaram a essência do sentido da resposta", como afirmam Lefèvre \& 
Lefèvre (2003). A partir da construção do DSC criamos categorias de análise, apresentadas a seguir:

\section{Concepções sobre a Etiologia da Tuberculose}

As informações que constituíram esta categoria foram coletadas a partir da primeira pergunta do roteiro de entrevista: "O que você sabe sobre a tuberculose?"

Podemos perceber que nas falas relacionadas a esta pergunta, a maioria dos pacientes confundiu etiologia com a forma de transmissão da doença. Percebemos que os pacientes tiveram muitas dificuldades para descrever sua patologia.

Houve respostas que associaram a TB a um vírus ou a fungo, ou a picada de mosquito e/ou a cuspir sangue, mostrando desta forma o desconhecimento dos pacientes sobre a etiologia de sua doença. O discurso predominante entre os entrevistados foi relacionado à forma de transmissão da TB e às manifestações clínicas de sinais e sintomas.

DSC

"Sei que é contagioso, que tem que tratar... que leva à morte. No fundo no fundo eu não sei o que que é, mas sei que geralmente é pulmonar, né? Eu sei só dos sintomas... dos sintomas que eu passei a sentir: falta de apetite, comecei a perder peso, cuspir sangue na calçada, febre, indisposição e muito cansaço. Enfraquece os ossos, que ela ultrapassa do grau da doença e que ela pode matar. Sei que é um fungo, que é um vírus que vai comendo nossos pulmões aos poucos minando nossa resistência. Sei que é transmitida pelo ar, pela picada do mosquito”.

Podemos observar que há algumas falas que correspondem ao discurso médico, enquanto que outras não. Os pacientes expressaram nas falas o saber popular do que seja a doença mediante as suas vivências e experiências de vida. Esta observação também foi evidenciada e descrita no trabalho de Souza, Natal \& Rozemberg (2005).

Percebe-se que há desinformação na maioria dos pacientes entrevistados. A população em geral, no decorrer da vida, recebe informações sociais, culturais e familiares nas escolas e através dos meios de comunicação em massa. As informações recebidas podem ter uma carga de temor, estigma e também crenças, que são disseminadas pela comunidade (Natal et al., 1999). 
Outro problema que observamos nestes pacientes é a baixa escolaridade, onde a maioria apresenta menos de oito anos de instrução. Este problema interfere no entendimento da linguagem médica (terminologia técnica) e, portanto a compreensão das orientações durante a consulta fica comprometida.

É importante que as orientações e as prescrições dos medicamentos sejam realizadas de maneira simples e clara, tanto falada quanto escrita. De fato, percebe-se que os pacientes só assimilam parte das orientações técnicas faladas pelos profissionais de saúde (Natal, et al., 1999; Sbarbaro, 2002).

\section{Sobre a Transmissão da Tuberculose}

Também em relação à pergunta "Como se adquire a tuberculose?" Percebemos que a maioria dos entrevistados respondeu de forma parcialmente correta. Quando começavam a explicar, apareceram alguns mitos que ainda nos dias de hoje estão presentes na questão da TB.

Há a ideia de que a transmissão seja devido à exposição ao sereno ou a de ficar trancado dentro de casa. Mas, de fato, se a pessoa ficar trancada dentro de casa de forma aglomerada, sem condições mínimas de ventilação é uma questão problemática e propensa as doenças, principalmente às transmissíveis pelo ar. Cabe ao profissional de saúde e educador trabalhar com os conceitos presentes na comunidade aprimorando-os através de estratégias de ensino, com uma linguagem simples e clara. Outra percepção sobre a transmissão pela TB é associada à ingestão de "Dhréa" (bebida alcoólica) ou ao uso de cocaína. Percebe-se que há uma confusão quanto à associação da bebida alcoólica em relação à transmissão da $\mathrm{TB}$, mas podemos considerar que o alcoolismo é um dos que fatores associados à TB.

Em uma das falas houve um relato em que se afirmou que a transmissão da TB acontece de geração em geração, quer dizer, através da genética familiar. O fato do indivíduo bacilífero dentro de casa, em contato com seus familiares, pode transmitir a doença através do ar, fica configurado para algumas pessoas como um fator genético.

De acordo com Gonçalves:

Até o final do século XIX, a população acreditava que a tuberculose estava intimamente ligada à hereditariedade e às condições de vida. Essa idéia foi devido ao adoecimento de várias pessoas da mesma família, o que levava os médicos da época considerar a tuberculose como hereditária (Gonçalves, 2000; p. 306). 
DSC:

"Muito sereno, você ficar trancado dentro de casa, num saí, num pegar um sol, né? Tomar muito gelado também provoca, cigarro, pela água... Beber em copo de bar, comer em talheres de outra pessoa... Não sei, os outros comenta que deve sê de geração de familiares, deve ser isso né... Eu acho que é isso. Bom, eu peguei com Drhéa e cocaína. Drhéa é um conhaque muito forte, foi porque eu bebia e fumava na rua o cigarro da boca de todo mundo."

Em relação à transmissão da tuberculose, observamos que entre as concepções relatadas, há o pensamento da necessidade de separar os copos, os talheres e demais utensílios domésticos como possível precaução para evitar a transmissão da doença (OPAS, 2002). No entanto, sabe-se que não há na literatura científica este tipo de abordagem e nenhuma pesquisa que tenha comprovado experimentalmente que há bacilo nos utensílios usados por pacientes com TB em fase bacilífera.

De modo geral, percebe-se através das falas dos pacientes a necessidade de trabalhar a educação em saúde baseada no diálogo, na troca de saberes, de forma a favorecer a compreensão mútua entre os saberes técnico e popular, levando a possíveis mudanças no entendimento das doenças e de sua prevenção (Souza, Natal \& Rosemberg, 2005).

\section{O Tratamento da Tuberculose visto pelos Pacientes}

Esta categoria está relacionada ao tratamento da TB. A pergunta utilizada para análise das respostas foi a seguinte: "O que você poderia falar sobre o tratamento da tuberculose?”. De acordo com as falas dos pacientes, há uma aceitação do tratamento por alguns relatando que é "bom e maravilhoso e que os sinais e sintomas da doença desaparecem rápido". "Que é necessário que se tenha força de vontade para terminar o tratamento". As queixas predominantes nas falas dos pacientes foram associadas aos efeitos adversos das medicações, à quantidade de comprimidos e cápsulas e à duração do tratamento. Dos 17 entrevistados, $14(82,4 \%)$ são soropositivos para o HIV e o cotidiano destes pacientes costuma ser a ingestão dos anti-retrovirais. Este fato pode ocasionar ainda mais efeitos adversos devido ao acréscimo dos anti-retrovirais aos tuberculostáticos, pois apesar de haver uma resposta terapêutica satisfatória, as sensações desagradáveis são fatores limitantes dos tratamentos que envolvem muitas drogas e são longos (Rolla et al., 2006). Um paciente mostrou esses aspectos em sua 
fala: "o tratamento da tuberculose é simples, acho simples. Não tem dificuldade... Eu achei um pouco forte, começaram o enjôo... O tratamento é adequado, é bom...”.

O tratamento é eficaz e logo nos primeiros dois e três meses de tratamento há uma melhora do quadro geral do paciente, mas isso não quer dizer que o indivíduo está curado. O tratamento com os tuberculostáticos leva no mínimo seis meses, passando para nove a doze meses quando o paciente retorna com história de abandono, como preconizado pelo Ministério da Saúde (2004).

Reforçando o relato acima Natal descreve em seu estudo que:

\begin{abstract}
"Os abandonos aconteceram predominantemente na primeira metade do tratamento e que vários autores associam o abandono nesta época devido à melhora clínica do doente. Foi verificado, em outros estudos, que a aderência caiu no segundo mês de tratamento. Diante de uma melhora clínica, o doente, se não é estimulado, modifica suas prioridades que passam a ser outras" (Natal et al, 1999 p. 72).
\end{abstract}

Há uma necessidade de realizar estratégias que possam estimular e criar um vínculo profissional de saúde-paciente e paciente-serviço público, para aumentar a adesão do paciente ao tratamento. Desta forma, há a possibilidade de identificar os possíveis casos de irregularidade do tratamento e o conseqüente abandono, dando condições aos profissionais de saúde de otimizar os recursos humanos, materiais e financeiros disponíveis para trabalhar.

DSC

"É horrível porque a gente tem que tomar remédio em jejum, entendeu? Começa a urinar vermelho... O que me levou a abandonar foi isso porque eu achei que já estava legal, né... Com os três meses tudo começou a voltar ao normal. ... Aí com dois ou três meses de tratamento me sentia melhor... Parei de ter cansaço, comecei a ganhar peso de novo... Achei que já tivesse bom... O tratamento é muito demorado né... Seis meses... Tinha que ser menos, ela só cura depois de seis meses né..? Essa minha agora, eu acho, que é a quinta vez, tive cinco tuberculose, afetou o fígado ... Porque com os retrovirais e mais o remédio da tuberculose dá uma sobrecarga do metabolismo que a pessoa se torna uma coisa de tantos remédio pra tomar que se torna um certo desânimo, entendeu?"

Percebe-se no discurso do coletivo que há predominantemente as queixas relacionadas ao uso de tuberculostáticos manifestando-se através dos efeitos adversos. 
Quando há reações leves, normalmente o médico prescreve medicações que amenizam os efeitos adversos, dando continuidade ao tratamento. Nas reações moderadas, o médico pode suspender o tratamento ou trocar o esquema em uso. Quando há a ocorrência de reações graves, o paciente é internado e tem o tratamento suspenso até a melhora do quadro clínico geral. Posteriormente, há avaliação médica para prescrever os tuberculostáticos de segunda escolha ou tentar re-introduzir as mesmas drogas que muitas vezes passam a ser bem toleradas (Ministério da Saúde, 2004).

\section{Motivos que levaram os Pacientes ao Abandono do Tratamento da Tuberculose}

A categoria que analisaremos corresponde à seguinte questão: "O que levou você a desistir de seu tratamento da tuberculose?" Esta pergunta possibilitou aos pacientes relatarem os seus motivos do abandono do tratamento da TB.

Os problemas relatados pelos pacientes evidenciam diversas situações de questões familiares, de ordem financeira, as queixas sobre os efeitos adversos dos tuberculostáticos aparecem novamente nesta categoria, além da discriminação e do estigma associada à TB.

O aspecto sócio-econômico é um fator que na maioria das vezes leva os pacientes a desistirem do tratamento da TB. Os gastos com alimentação e de transporte para ir às consultas e para realizar os exames, uma vez que os pacientes com TB não têm direito à gratuidade de transporte.

DSC

"Era recente eu tinha descoberto que tinha o HIV, muito, muito probrema de cabeça, muita coisa... Não aceitava muito bem aquilo que eu tinha. A quantidade de remédio. Os remédios é muito forte, fica muito enjoado... Foi essa quantidade de remédio que eu tomo pro HIV juntou muita coisa, ai comecei a enjoar. Eu não suportava... Aí fiquei mais de um mês sem tomar nada. ... Eu desanimei porque toda vez que tinha que vim e tirar sangue. E tirava uns cinco litros de sangue e nunca o sangue dava, pelo amor de Deus e eu desisti mais por causa disso, porque toda vez que eu vinha tinha que tirar sangue... Abate muito a pessoa né, e muita gente sabe que a maioria que pega tuberculose é porque tá com o sintoma do HIV, não é?

De acordo com as falas dos entrevistados, percebe-se que são vários os motivos que levam os pacientes a abandonar o tratamento da TB. Dentre eles, a presença de 
sensações corporais desagradáveis, como os efeitos adversos à medicação, e a falsa crença de que pelo fato de não mais apresentar sinais e sintomas da doença, acreditam que já estão curados e por isso abandonam o tratamento, mostrando desta forma que a concepção de doença está intimamente ligada à presença destas características sintomatológicas.

Outro problema é em relação aos pacientes infectados pelo HIV que já desenvolveram a forma mais avançada desta doença. Nas falas de alguns pacientes percebemos o estigma e a discriminação que são os maiores obstáculos para medidas efetivas de educação em saúde e prevenção no âmbito da co-infecção TB/HIV. O medo da discriminação pode impedir que estes pacientes busquem tratamento contra a AIDS e conseqüentemente da TB, como o temor de que as suas situações sorológicas em relação ao HIV sejam publicamente conhecidas. As pessoas infectadas ou supostamente infectadas pelo HIV (pela aparência física) podem ser rejeitadas pelos serviços de saúde, privadas de moradia e emprego, evitadas por amigos e colegas, descobertas por seguros de saúde ou recusadas para ter entradas em países estrangeiros (Castilho, 2002).

No entanto, na questão da TB, existem fatores que influenciam no abandono do tratamento que estão relacionados ao serviço de saúde. Segundo Deheinzelin e colaboradores (1996), o estreitamento do contato médico-paciente e a redução do tempo de espera para a consulta estão associados a uma boa adesão. Muitos pacientes se queixam do longo tempo de espera dentro do serviço de saúde, desestimulando a retornar e talvez até mesmo a dar continuidade ao tratamento, ainda mais para àqueles que se encontram assintomáticos logo nos primeiros meses do início da terapia medicamentosa (Natal et al., 1999).

No LAPCLIN TB do IPEC o agendamento das consultas tem hora marcada, com um espaço de meia hora entre as consultas, mas ainda assim presenciamos algumas reclamações dos pacientes.

Outros relatos que permanecem nas falas dos entrevistados referem-se às queixas sobre a quantidade de medicamentos e os efeitos adversos. Segundo Gonçalves et al. (1999) relatam que os pacientes abrem as cápsulas para investigar o conteúdo e a cor do medicamento; a cor vermelha, neste caso da rifampicina, é associada à potência/força do medicamento, significando para "eles" que é um remédio mais forte. Desta forma, começa haver uma rejeição por parte dos pacientes ao medicamento por eles considerado ruim ou forte. Foi observado no estudo realizado por estes autores que, 
quanto maior a irregularidade na ingestão dos medicamentos, menor a probabilidade de que cessem os efeitos indesejáveis dos tuberculostáticos.

\section{Possíveis Estratégias para Diminuir o Abandono do Tratamento}

Com relação à categoria "O que você acha que poderia mudar para facilitar o seu tratamento?" Os pacientes puderam expor situações que os incomodam e sugerir as possíveis estratégias de mudanças. As falas dos entrevistados, em alguns momentos, aparecem repetidas em relação às questões sócio-econômicas já mencionadas nas respostas anteriores.

Percebemos que as colocações predominantes nas falas dos pacientes estão associadas aos medicamentos. Destacamos como Ideias Centrais nesta categoria: Diminuir a quantidade de remédios, diminuir o tempo de tratamento. Ouvimos muitas queixas quanto ao uso de tuberculostáticos, a quantidade de cápsulas e comprimidos que são ingeridos por dia e em conseqüência disso os vários relatos sobre os efeitos adversos que os medicamentos ocasionam nestes pacientes. Alguns pacientes relatam que além dos problemas com os medicamentos, acrescentam-se os de ordem financeira e familiar, como a falta de emprego e a discriminação dentro de casa.

DSC

"Se eu arrumasse um emprego, digno... Se pudesse diminui os remédios que eu tomo que é muita quantidade, é muita quantidade... é... chega a ter uns 14, 15 comprimidos por dia, é por dia. ... Vou te falar, por mim é o remédio em jejum, eu não suporto!!! Não

tomar em jejum. É... Diminuir o tempo de tratamento.... Que eu não podia pegar nas criança, não podia isso, não podia aquilo e por isso que eu larguei também foi mais por causa disso. Eu tive muita discriminação. Muitas vezes já abandonei a consulta porque o médico demorou me atender. Tem que ser mais rápido a consulta. A passagem pra mim ir e vir. Desisti porque eu vivia na rua e não tinha cabeça”.

Em suas narrativas, podemos identificar as dificuldades financeiras que os pacientes têm; o desejo de ter um emprego, de ter dignidade. Junto a esta questão tem a presença de discriminação ainda enraizada na sociedade e entre os familiares, tornando cada vez mais desestimulante para os pacientes aderir ao tratamento.

Outra questão abordada foi em relação à demora no atendimento, ficando algumas horas na sala de espera, o que ocasionou e levou à desistência das consultas. 
Alguns pacientes chegaram a sugerir uma consulta mais rápida. As consultas realizadas no LAPCLIN TB do IPEC são minuciosas, os pacientes são examinados cuidadosamente, caracterizando um atendimento que é de qualidade dentro das boas práticas clínicas. No entanto, a sugestão dos pacientes é de que as consultas sejam mais curtas, mostrando o desafio de compatibilizar uma consulta rápida e a qualidade do atendimento.

Teklu (1984) e Natal et al. (1999) afirmam que:

“... fatores ligados ao serviço, citados na literatura como associados ao abandono, são o tempo de demora no atendimento e necessidade do uso de mais um transporte coletivo. Quanto maior a espera para o atendimento em um ambulatório, mais elevada a proporção de ausentes na consultas seguintes (Teklu, 1984, p. 18; Natal et al., 1999, p. 76).

A equipe multidisciplinar faz a diferença no atendimento aos pacientes, desta forma diminuindo o abandono de tratamento e proporcionando um atendimento mais humanizado e holístico. A interação entre os profissionais descentraliza as informações e qualificam o atendimento, diminuindo a falta de comunicação e as dúvidas.

\section{Sugestões dos Pacientes para a Sala de Espera do Programa de Tuberculose}

A última pergunta que foi: "O que você gostaria que tivesse na sala de espera?" Neste momento obtivemos várias sugestões que poderão ser implementadas para mudar a sala de espera do LAPCLIN TB, como: um vídeo que fale sobre a TB, uma palestra, folders e cartilhas. As sugestões apareceram de forma diversificada pelo coletivo, mostrando desta forma que estão interessados que tenha na sala de espera atividades de educação em saúde, onde os pacientes e familiares possam participar e interagir nas discussões.

DSC

"Gostaria que tivesse uma palestra até à hora da consulta da médica. Tinha que ter uma pessoa pra explicar, né... Um vídeo a respeito disso entendeu, explicando as família, os parente como é que é, o acompanhante que tivesse um doente e o próprio doente mesmo que é muito importante, entendeu? Mais folhetos sobre a doença, mais explicativo assim... mostrando o que a doença pode causar, a parada do remédio, mostrando assim...o que a doença faz no nosso organismo realmente, como nossos pulmões ficam, o que é a tuberculose disseminada, essas coisas que agente não tem 
noção. Ah e bastante quadro de aviso, escrito, forma de precaução, forma de como evitar o contágio das pessoas.”

Podemos destacar de acordo com o discurso analisado que há uma preocupação e desejo dos pacientes de ter conhecimento sobre sua doença, de participar de uma palestra e poder tirar suas dúvidas. Durante a realização das entrevistas, pudemos observar na maioria dos pacientes uma satisfação de poder expor, de ter um profissional da saúde ouvindo suas queixas, dores, angústias, suas dúvidas, suas sugestões e expectativas e de participar desse processo de construção e de melhor atendimento pelo LAPCLIN TB. Alguns pacientes se mostraram felizes por estarmos preocupados em identificar os fatores que os influenciaram a abandonar o tratamento da $\mathrm{TB}$, da iniciativa da realização deste estudo.

Em 2005, foi realizado um estudo no IPEC abordando questões sobre a epidemiologia e prevenção das doenças infecciosas e parasitárias direcionadas a pacientes do projeto de prevenção em tuberculose e seus familiares e amigos. Este trabalho proporcionou aos participantes a construção de um cotidiano permeado pela humanização, permitindo a este grupo trocar e construir conhecimentos, elaborando propostas, redefinindo ou anulando normas, construindo outras possibilidades de fazer o processo educativo (Souza et al., 2005).

As respostas correspondentes a esta categoria mostraram a satisfação dos pacientes em participar deste processo de construção dialógica. Fato este que deveria ser estimulado pelas autoridades e gestores da saúde em geral, agindo de forma objetiva e otimizando os recursos financeiros disponíveis para maior participação da comunidade e interesse como um todo na prevenção e na educação em saúde. Tal afirmativa é discutida e apresentada por Briceño-Léon (1996), que enfatiza que a educação em saúde, como prática social, baseada no diálogo, ou seja, na troca de saberes, favorece a compreensão da relação entre saberes técnicos e leigos no processo saúde-doença.

\section{Estratégias de Educação em Saúde}

Promover estratégias de educação em saúde requer ações que envolvam as coletividades em geral e a família, de forma que provoquem mudanças de comportamento de atitudes capazes de beneficiar a saúde da população e melhorar a auto-estima dos pacientes. A estratégia de educação em saúde, oferecida aos indivíduos, grupos e à coletividade, possibilita um comportamento positivo para a saúde, 
permitindo o desenvolvimento de maior controle sobre os fatores que a determinam; favorece uma vida mais saudável e melhora a auto-estima (Candeias, 1997). É imprescindível que os pacientes sejam participantes, com liberdade e direito de tomar decisões conscientes sobre sua saúde.

As estratégias de educação em saúde devem permitir o convívio e interações enriquecedoras entre os participantes e devem ser planejadas de acordo com as necessidades levantadas. Esse levantamento foi possível devido à realização das entrevistas onde identificamos as necessidades dos pacientes de obterem informações sobre sua doença, do seu tratamento e o conhecimento dos problemas decorrentes da interrupção do tratamento, etc.

DSC

"Talvez um vídeo explicando sobre a doença, sobre o remédio, sobre o tratamento, quanto tempo leva, se eu ia melhorar. O que a doença pode causar, a parada do remédio, mostrando assim... o que a doença faz no nosso organismo realmente, como nossos pulmões ficam, o que é a tuberculose disseminada, essas coisas que agente não tem noção. Eu acho que pelo menos daria uma significação assim... pra nossa mente saber o que é que agente está tratando”.

Identificamos no grupo sob estudo o interesse de compartilhar saberes através do estabelecimento da relação enfermeiro-paciente ou pesquisador/educador-entrevistador, fato importante para trabalhar a educação em saúde.

\section{Considerações Finais}

Este estudo almejou identificar os fatores que influenciaram os pacientes a abandonarem o tratamento da tuberculose e em conseqüência disso, a implementação de um Programa de Educação em Saúde direcionado a esta clientela, para prevenir o abandono e estimular a adesão ao tratamento.

Verificamos que o perfil clínico-epidemiológico dos pacientes entrevistados é de indivíduos com poucos anos de escolaridade, com baixa ou nenhuma renda mensal de até dois salários-mínimos, com história pregressa de TB, e uma freqüência relevante da infecção pela TB/HIV. Esses relatos justificam não só o estabelecimento de ações no setor de saúde, mas também em outros setores de no âmbito sócio-econômico e político, que reduzam a pobreza da população. 
Durante a elaboração deste trabalho detectamos algumas necessidades que já estão sendo incorporadas na rotina do ambulatório do IPEC, como estratégias de captação de pacientes que abandonaram o tratamento da TB através da consulta de adesão a estes pacientes.

Esta consulta prioriza pacientes que apresentam fatores de risco para abandonar em algum momento o tratamento da TB. De acordo com a literatura, são aqueles que têm: história de abandono de tratamento, tabagismo, alcoolismo, faltosos às consultas agendadas anteriormente e com recidiva de TB (Braga, Hijar \& Procópio, 2004; Ministério da Saúde, 2004; Carvalho et al., 2006).

Durante a realização da consulta de adesão e na sala de espera utilizamos como recurso visual e simbólico alguns comprimidos e cápsulas que fazem parte do tratamento da $\mathrm{TB}$, material didático mostrando os pulmões acometidos pela $\mathrm{TB}$ com a intenção de tirar as dúvidas e discutir os potenciais problemas decorrentes do abandono de tratamento.

Observamos que as atividades educativas que vêm sendo realizadas na sala de espera são oportunidades excelentes de estabelecer uma relação com os pacientes, considerando que qualquer intervenção em saúde implica uma ação pedagógica.

Na prática, observamos as dificuldades dos profissionais de saúde desenvolver a educação em saúde visto que muitas vezes não conseguem ter uma linguagem acessível (não técnica) para conversar com os pacientes. Este fato revela ser um ponto fundamental a ser refletido, pois se evidencia o distanciamento do diálogo do profissional de saúde frente ao paciente, assim contrariando suas necessidades. É necessário que os profissionais de saúde possam rever as suas práticas educativas, aprendendo uma linguagem popular, com possibilidades de diálogo e escuta sensível em relação aos seus pacientes.

Cabe ressaltar que a realização desta pesquisa vem proporcionando estímulo e motivação não só nos profissionais de saúde do IPEC como também nos pacientes que estão sendo acompanhados no LAPCLIN TB.

Esperamos que novas iniciativas em ensino não formal sejam implementadas para contribuir no direcionamento de ações de Saúde Pública, mais especificamente no controle da TB, objeto de nosso trabalho. 


\section{Referências Bibliográficas}

ALBUQUERQUE, M.F.M. et al. Fatores prognósticos para o desfecho do tratamento da tuberculose pulmonar em Recife, Pernambuco, Brasil. Rev Panam Salud Publica, 9 (6): 368-375. Junho, 2001.

BERGEL, F.S.; GOUVEIA, N. Frequent return as a novel strategy for tuberculosis treatment adherence. Rev. Saúde Pública, 39 (6): 898-905. Dezembro, 2005.

BRAGA, J.U.; HIJJAR, M.; PROCOPIO, M. Relatório de avaliação da situação epidemiológica da tuberculose no Brasil, 1990-2002 (resultados preliminares). Rio de Janeiro: Centro de Referência Professor Hélio Fraga; 2004.

BRICEÑO-LÉON, R. Siete tesis sobre la educacion sanitária para la participación comunitária. Cadernos de Saúde Pública, 12 (1): 7-30. Janeiro-março, 1996.

CANDEIAS, Nelly Martins Ferreira. Conceitos de educação e de promoção em saúde: mudanças individuais e mudanças organizacionais. Rev. Saúde Pública, 31 (2): 209213. Abril, 1997.

CARVALHO, S.S.; ROLLA, V.C.; COSTA, M.J.M.; VELASQUE, L.S. Risk Factors for Tuberculosis treatment Abandoning in the Rio de Janeiro Tuberculosis Program (2000-2004). In: International AIDS Conference 2006. Toronto. Anais da International AIDS Conference, 67-67, 2006.

CASTILHO, E.A. Stigma and discrimination: "live and let live". World AIDS

Campaigen 2002-2003. Campaigen, 2002. Disponível em: URL: http://www.unaids.org/wac/2002/index.html. Acessado em 01 de fevereiro de 2008.

DEHEINZELIN, D.; TAKAGAKI, T.Y.; SARTORI, A.M.C.; LEITE, OH.M. Fatores preditivos de abandono de tratamento por pacientes com tuberculose. Revista do Hospital das Clínicas Faculdade de Medicina, 51(4): 131-135, 1996

DROBNIEWSKI, F.A.; BALABANOVA, Y.M. The diagnosis and management of multiple-drug-resistant tuberculosis at the beginning of the new millenium. International Journal of Infeccious Diseases, 6 (Supplement 1), p. S21-S31. Março, 2002.

LEFÈVRE, F.; LEFÈVRE, A.M.C. O discurso do sujeito coletivo: um enfoque em pesquisa qualitativa (Desdobramentos). Caxias do Sul, RS: Editora EDUCS, 2003.

MINISTÉRIO DA SAÚDE. Fundação Nacional de Saúde. Controle da Tubeculose: Uma Proposta de Integração Ensino-Serviço, 5 $5^{\text {a }}$ ed. - Rio de Janeiro: FUNASA/CRPHF/SBPT, 236p, 2002. 
MINISTÉRIO DA SAÚDE. II Consenso Brasileiro de Tuberculose. Coordenação Nacional de Pneumologia Sanitária. Sociedade Brasileira de Pneumologia e Tisiologia. Jornal de Pneumologia; 30(1) S5-S20, 2004. Pp. 34-40

MINAYO, M.C.; DESLANDES, S.F.; GOMES, R. Pesquisa Social: Teoria, método e criatividade. Rio de Janeiro: Editora Vozes, 2002. P. 21

NATAL, S.; VALENT, J.; GERHARDT, G.; PENNA, M.P. Modelo de predição para o abandono do tratamento da tuberculose pulmonar. Boletim de Pneumologia Sanitária, 7(1): 65-78. Jan-jun, 1999.

NETTO, E.M., DYE, C., RAVIGLIONE, M.C. Progress in global tuberculosis control 1995-1996, with emphasis on 22 high-incidence countries. Global Monitoring and Surveillance Project. International Journal Tuberculosis Lung Diseases, 3(4): 310320. Abril, 1999.

NOGUEIRA, J.A, RUFFINO-NETTO, A; SCATENA, T.C.; VILLA, MY; ARCENCIO, R.; CARDOZO-GONZALES, R.I. Aspectos epidemiológicos da coinfecção tuberculose e vírus da imunodeficiência humana em Ribeirão Preto (SP), de 1998 a 2003. Jornal de Pneumologia, 32(6): 529-534. Dezembro, 2006.

ORGANIZAÇÃO PANAMERICANA DE SAÚDE. Sistemas de informação

geográfica em saúde: conceitos básicos. Brasília (DF): OPAS; 2002.

ROLLA, V.C.; VIEIRA, M.A.S.; PINTO, D.P.; LOURENÇO, MC.; DE JESUS, C.S.; GONÇALVES, M.M.; MORGADO, M.G.; FILHO, M.F.; WERNECK-BARROSO, E. Safety, efficacy and pharmacokinetics of ritonavir $400 \mathrm{mg} / \mathrm{saquinavir} 400 \mathrm{mg}$ twice dailly plus rifampicin combined therapy in HIV patients with tuberculosis. Clinical Drug Investigation, 26(8): 469-479. Outubro, 2006.

RUBEL, A.J.; GARRO, L.C. Social and Cultural Factors in the Successful Control of Tuberculosis. Public Health Reports, 107(6): 626-636, Dezembro, 1992.

RUFFINO-NETTO, A. Tuberculose: a calamidade negligenciada. Revista da Sociedade Brasileira de Medicina Tropical, 35(1): 51-58. Jan-fev, 2002.

SBARBARO, J.A. The treatment of tuberculosis: reflections on Siberia. International Journal Tuberculosis Lung Diseases, 6 (5): 375-7. Maio, 2002. Review.

SOUZA, C.T.V; NATAL, S.; ROZEMBERG, B. Comunicação sobre prevenção da tuberculose: perspectivas dos profissionais de saúde e pacientes em duas unidades assistenciais da Fundação Oswaldo Cruz, Rio de Janeiro. Revista da ABRAPEC, 5(1), 2005. 
SOUZA, K.R.S.; ROSEMBERG, B.; KELLY-SANTOS, A.; YASUDA, N.; SHARAPIN, M. O desenvolvimento compartilhado de impressos como estratégia de educação em saúde junto a trabalhadores de escola da rede pública do Estado do Rio de Janeiro. Caderno de Saúde Pública, 19(2): 495-504. Março-Abril, 2003

SUMARTOJO, E. When tuberculosis treatment fails: a social behavioral account of patient adherence. American Review of Respiratory Disease. 147(5):1311-2. Maio, 1993.

TANGÜIS, H.G.; CAYLÁ, J.A.; OLALLA, P.G.; JANSÁ, J.M.; BRUGAL, M.T. Factors predicting non-completion of tuberculosis treatment among HIV-infected patients in Barcelona (1987-1996). International Journal Tuberculosis Lung Diseases. 4(1): 55-60. Janeiro, 2000.

TEKLU, B. Reasons for failure in treatment of pulmonary tuberculosis in Ethiopians. Tubercle, 65(1):17-21. Março,1984.

WORLD HEALTH ORGANIZATION. Global tuberculosis control: surveillance, planning, financing. WHO report 2004. WHO/HTM/TB 2004.331. Geneva, Switzerland: WHO, 2004. 